\title{
Status of Atomic PNC: Experiment/Theory
}

\author{
W. R. Johnson \\ University of Notre Dame
}

\begin{abstract}
Atomic PNC measurements and calculations are reviewed with emphasis on the $6 s \rightarrow 7 s$ transition in cesium and the corresponding value of the weak charge $Q_{W}\left({ }^{133} \mathrm{Cs}\right)$.
\end{abstract}

\section{Introduction}

More than a quarter century ago, Bouchiat and Bouchiat [1] discovered that parity non-conservation (PNC) in atoms, described in the standard model of the electroweak interaction by exchange of $Z$ bosons between bound electrons and nuclear quarks, grows rapidly with nuclear charge, making it large enough to be measurable in some heavy atoms. A consequence of PNC is that atomic states are mixed with states of opposite parity; thereby violating Laporte's rule [2], and permitting nonvanishing electric-dipole matrix elements $E_{\mathrm{PNC}}$ between atomic states with the same nominal parity. The nuclear spin-independent part of the PNC interaction Hamiltonian, arising from the vector nucleon current, is

$$
H^{(1)}=\frac{G}{2 \sqrt{2}} \gamma_{5} Q_{W} \rho(r),
$$

where $Q_{W}$ is a conserved given by

$$
Q_{W}=-N+Z\left(1-4 s^{2}\right) \approx-N,
$$

with $s^{2}=\sin ^{2} \theta_{W} \approx 1 / 4$. In the above equation, $\rho(r)$ is a normalized density for the nuclear quarks; it is approximately the neutron density. Measurements of $E_{\mathrm{PNC}}$, when combined with suitable atomic structure calculations, permit one to obtain experimental values of $Q_{W}$, which are known to be sensitive to physics beyond the standard model $[3,4]$. In this regard, a $2.5 \sigma$ difference between the precise experimental value of the weak charge of ${ }^{133} \mathrm{Cs}$ reported by Wood et al. [5] and the value predicted by the standard model of the electroweak interaction was found several years ago by Bennett and Wieman [6]. This difference, being one of the two largest differences reported in the current particle data book [7], suggested the existence of a second neutral $Z^{\prime}$ boson [8-11]. The deviation could also be explained by four-fermion contact interactions with specific chiralities or by scalar leptoquarks which couple to the left-handed quarks [12]. In the present report, we give a brief review of PNC measurements and calculations for atoms and update the experimental value of the weak charge of ${ }^{133} \mathrm{Cs}$ in light of recent improvements in calculations of PNC matrix elements. We also review PNC experiments on other elements: proposed, underway, and completed.

\section{Optical Rotation}

In one important class of experiments, advantage is taken of the fact that atomic PNC leads to optical activity in monatomic gas vapors. The optical rotation of linearly polarized radiation, tuned to an E1-forbidden transition, is measured. From such 
Table 1: Optical rotation measurements of $R_{\phi}$ over the past decade.

\begin{tabular}{cclr}
\hline Element & Transition & \multicolumn{1}{c}{ Reference } & $10^{8} \times R_{\phi}$ \\
\hline${ }^{205} \mathrm{Tl}$ & ${ }^{2} P_{1 / 2}-{ }^{2} P_{3 / 2}$ & Edwards et al. [13] & $-15.33(45)$ \\
${ }^{205} \mathrm{Tl}$ & ${ }^{2} P_{1 / 2}-{ }^{2} P_{3 / 2}$ & Vetter et al. [14] & $-14.68(20)$ \\
${ }^{208} \mathrm{~Pb}$ & ${ }^{3} P_{0}-{ }^{3} P_{1}$ & Meekhof et al. [15] & $-9.86(12)$ \\
${ }^{208} \mathrm{~Pb}$ & ${ }^{3} P_{0}-{ }^{3} P_{1}$ & Edwards et al. [16] & $-9.80(33)$ \\
${ }^{209} \mathrm{Bi}$ & ${ }^{4} S_{3 / 2}-{ }^{2} D_{3 / 2}$ & Macpherson et al. [17] & $-10.12(20)$ \\
\hline \hline
\end{tabular}

a measurement, the ratio $R_{\phi}=\operatorname{Im}\left(E_{\mathrm{PNC}}\right) / M 1$ can be extracted, where $M 1$ is the magnetic-dipole amplitude for the transition. Precise measurements of $R_{\phi}$ have been carried out for the $6 p_{1 / 2}-6 p_{3 / 2}$ transition in ${ }^{205} \mathrm{Tl}$ in Oxford [13] and Seattle [14]; for the $6{ }^{3} P_{0} \rightarrow 6{ }^{3} P_{1}$ transition in ${ }^{208} \mathrm{~Pb}$ in Seattle [15] and Oxford [16]; and for the ${ }^{4} S_{3 / 2}-{ }^{2} D_{3 / 2}$ transition in ${ }^{209} \mathrm{Bi}$ in Oxford [17]. Values of $R_{\phi}$ from the more accurate optical rotation measurements carried out over the past decade are summarized in Table 1.

The experimental results for ${ }^{205} \mathrm{Tl}$ shown in the table formally contradict one another. Majumder and Tsai [18] suggested that the discrepancy could be due to different values of the ratio of $E 2$ to $M 1$ amplitudes used by the two groups. They re-measured this ratio and used it to re-scale the result of [13] and find

$$
R_{\phi}\left({ }^{205} \mathrm{Tl}\right)=-14.71(45) .
$$

Combining this value of $R_{\phi}$ with the most recent many-body calculation of the PNC amplitude in thallium [19] leads to

$$
Q_{W}^{\exp }\left({ }^{205} \mathrm{Tl}\right)=113(3) .
$$

The error is primarily due to uncertainties in the many-body calculation which is particularly difficult for a heavy atoms, such as those in Table 1, with two or more valence electrons. The experimental value for thallium can be compared with the value predicted by the standard model,

$$
Q_{W}^{\mathrm{SM}}\left({ }^{205} \mathrm{Tl}\right)=116(1) .
$$

The experimental value is consistent with the standard model; however, if the error in the many-body calculation, which dominates that in the experimental weak charge, were reduced by a factor of 3 , the comparison would provide a really critical test of the standard model.

Cronin et al. [20] suggested that the $6 p_{1 / 2} \rightarrow 6 p_{3 / 2}$ PNC measurement could be improved using the electromagnetically induced transparency (EIT) [21] of thallium vapor. The proposed method, which was used in [20] to obtain the ratio $E 2 / M 1$ for the $6 p_{1 / 2} \rightarrow 6 p_{3 / 2}$ transition, should permit one to obtain sub-Doppler resolution for the optical rotation as well as improved background subtraction. More details of the EIT measurement in thallium can be found at the website [22]. 
Table 2: Evolving values of $R_{\text {Stark }}=(\mathrm{mV} / \mathrm{cm})$ for cesium.

\begin{tabular}{ccccc}
\hline Element & Transition & References & $\mathrm{R}_{4-3}$ & $\mathrm{R}_{3-4}$ \\
\hline${ }^{133} \mathrm{Cs}$ & $6 s_{1 / 2}-7 s_{1 / 2}$ & Bouchiat et al. [28] & $-1.5(2)$ & $-1.5(2)$ \\
${ }^{133} \mathrm{Cs}$ & $6 s_{1 / 2}-7 s_{1 / 2}$ & Wieman and Gilbert [29] & $-1.64(5)$ & $-1.51(5)$ \\
${ }^{133} \mathrm{Cs}$ & $6 s_{1 / 2}-7 s_{1 / 2}$ & Wood et al. [5] & $-1.635(8)$ & $-1.558(8)$ \\
\hline \hline
\end{tabular}

\section{Stark Interference}

In a second class of experiments [23, 24], a laser excites an E1-forbidden transition in an atomic beam in a presence of electric $E$ and magnetic $B$ fields. The quantity measured is the component of the transition rate arising from the interference between $E_{\mathrm{PNC}}$ and the Stark amplitude $\beta E$ is then used to determine the ratio $R_{\text {Stark }}=$ $\operatorname{Im}\left(E_{\mathrm{PNC}}\right) / \beta$. The quantity $\beta$ is the vector part of the Stark-induced polarizability for the transition and can be measured separately. A variant of this method has been used by Drell and Commins in $[25,26]$ to determine $R_{\text {Stark }}$ for the $6 p_{1 / 2} \rightarrow 7 p_{1 / 2}$ transition in ${ }^{205} \mathrm{Tl}$; later, $\beta$ for this transition was measured [27].

Stark interference has also been used over the past two decades to measure $R_{\text {Stark }}$ for the $6 s \rightarrow 7 s$ transition in ${ }^{133} \mathrm{Cs}$. The earliest of these were the $10 \%$ measurements by the Paris group [28]. These were followed by $2 \%$ measurements by the Boulder group [29] and, five years ago, $0.3 \%$ measurements by the Boulder group [5]. This evolution is shown in Table 2 , where we list values $R_{4-3}$ for $F=4 \rightarrow F=3$ transition and $R_{3-4}$ for the $F=3 \rightarrow F=4$ transition.

The contribution to $R_{\text {Stark }}$ from the vector current, obtained from the values on the last row of Table 2 , is $-1.593 \pm 0.006$. Combining this value with the accurate value of $\beta=27.024 \pm(0.043)_{\exp } \pm(0.026)_{\text {th }}$ obtained in [6], leads to

$$
10^{11} \times \operatorname{Im}\left[E_{\mathrm{PNC}}(6 s \rightarrow 7 s)\right]=-0.8374 \pm(0.0031)_{\exp } \pm(0.0021)_{\mathrm{th}} .
$$

This experimental value of the PNC amplitude must be combined with many-body calculations to obtain the weak charge of ${ }^{133} Q_{W}$.

The most recent many-body calculation of the PNC amplitude for cesium was carried out by Dzuba et al. [30] using a method referred to as "perturbation theory in the screened Coulomb interaction" (PTSCI) in which important classes of many-body diagrams are summed to all orders. The atomic structure calculations lead to a value in precise agreement with the value obtained by Blundell et al. [31] using a linearized single-double coupled-cluster (SDCC) method. The values are:

$$
\begin{aligned}
E_{\mathrm{PNC}}= & 0.908(5), & & (\mathrm{PTSCI}) \\
& 0.909(4), & & (\mathrm{SDCC})
\end{aligned}
$$

in units - i e $a_{0} Q_{W} / N \times 10^{-11}$. (We omit the Breit correction that was originally included in [31] and use the error estimate for that calculation given in [6].) Combining the calculations and the measurements, we find

$$
Q_{W}^{\exp }\left({ }^{133} \mathrm{Cs}\right)=-71.90(48) .
$$


As discussed in the introduction, this value disagrees by $2.5 \sigma$ with the standard mode value [7]

$$
Q_{W}^{\mathrm{SM}}\left({ }^{133} \mathrm{Cs}\right)=-73.09(3) .
$$

Particle physicists try to understand this disagreement in terms of a second $Z^{\prime}$ boson or other exotic mechanisms. The disagreement has also motivated atomic physicists to re-examine small corrections to the atomic structure calculations.

Indeed, Breit corrections to the PNC amplitude, which were ignored in [32] and underestimated in [31], decrease the size of the calculated PNC amplitude by $0.6 \%$ as shown by Derevianko [33] and confirmed in [34-36], reducing the difference with the standard model.

Radiative corrections to PNC matrix elements $\left\langle w\left|H_{\mathrm{PNC}}\right| v\right\rangle$ in the strong Coulomb field of a highly-charged one-electron ion were considered by Bednyakov et al. [37]. These corrections can be decomposed into two parts, radiative corrections to the operator $H_{\mathrm{PNC}}$, and radiative corrections to the wave functions $|v\rangle$ and $|w\rangle$. The former corrections were evaluated for ${ }^{133} \mathrm{Cs}$ in Refs. [38-42] and are already included in the standard model value $Q_{W}^{\mathrm{SM}}\left({ }^{133} \mathrm{Cs}\right)$. The part of the wave function radiative correction arising from vacuum-polarization in the nuclear Coulomb field was found to increase the size of $Q_{W}^{\exp }\left({ }^{133} \mathrm{Cs}\right)$ by $0.4 \%$ and partially cancel the Breit correction $[43,44]$. The remaining "wave-function" radiative correction is that arising from the photon vertex. Milstein and Sushkov [44] find that this vertex contribution changes the weak charge of ${ }^{133} \mathrm{Cs}$ by less than $0.1 \%$, while Kuchiev and Flambaum [45] contend that this term decreases the weak charge by $0.7 \%$.

Finally, the "nuclear skin" correction, which accounts for the fact that the neutrons are the source of the weak interaction whereas the more accurately known proton charge distribution is used for $\rho(r)$ in the PNC Hamiltonian, has been examined in [46-48] and found to decrease the size of the $Q_{W}\left({ }^{133} \mathrm{Cs}\right)$ by a further 0.1 to $0.2 \%$. Taking into account the Breit, radiative and "skin" corrections, there remains either a $2.2 \sigma$ or $1.0 \sigma$ difference with the standard model, depending on the value used for the wave-function vertex radiative correction!

A new PNC experiment aimed at confirming the Boulder result is underway at l'Ècole Normal Supérieur [49-51]. This experiment is the first of its kind conducted using a sapphire cesium vapor cell. The experimental configuration consists of two collinear laser beams, pump and probe, and a $2 \mathrm{kV} / \mathrm{cm} E$ field in the same direction. The attractive features of this pump-probe experiment are: i) it provides a direct measurement of the PV asymmetry at the output of polarimeter, ii) the calibration is lineshape independent (there is no $B$ field), iii) Stark-M1 interference is absent in a longitudinal field configuration, hence a potential source of systematics is suppressed, and iv) the right-left asymmetry is amplified when the probe beam propagates through an optically thick vapor. In view this new experiment, a similar effort on the theoretical side in evaluating the vertex wave-function radiative corrections is surely warranted.

\section{Other Important PNC Studies}

In Table 3, we list several other atoms for which measurements have been made or are being contemplated. Let us go through these cases in order: 
Table 3: Other atoms where PNC is studied.

\begin{tabular}{lll}
\hline Atom & \multicolumn{1}{c}{ Transition } & Group \\
\hline $\mathrm{Fr}$ & $7 S_{1 / 2} \rightarrow 8 S_{1 / 2}$ & Stony Brook \\
$\mathrm{Yb}$ & $\left(6 s^{2}\right)^{1} S_{0} \rightarrow(6 s 5 d)^{3} D_{1}$ & Berkeley \\
$\mathrm{Yb}$ & $(6 s 6 p)^{3} P_{0} \rightarrow(6 s 6 p)^{3} P_{1}$ & Berkeley \\
$\mathrm{Ba}^{+}$ & $6 S_{1 / 2} \rightarrow 5 D_{3 / 2}$ & Seattle \\
$\mathrm{Dy}$ & $\left(4 f^{10} 5 d 6 s\right)[10] \rightarrow\left(4 f^{9} 5 d^{2} 6 s\right)[10]$ & Berkeley \\
$\mathrm{Sm}$ & $\left(4 f^{6} 6 s^{2}\right)^{7} F_{J} \rightarrow\left(4 f^{6} 6 s^{2}\right)^{5} D_{J^{\prime}}$ & Oxford \\
\hline \hline
\end{tabular}

\subsection{Francium $(Z=87)$}

Francium is the heaviest alkali atom and shares with cesium the simplicity of having a single valence electron outside a closed core. The PNC matrix element $E_{\mathrm{PNC}}(\mathrm{Fr})\left[7 s_{1 / 2} \rightarrow\right.$ $\left.8 s_{1 / 2}\right]$ is 15 times larger than the corresponding $6 s_{1 / 2} \rightarrow 7 s_{1 / 2}$ matrix element in cesium. A program to measure PNC in francium has been underway at the State University of New York at Stony Brook for the past decade. Francium isotopes are produced by bombarding a gold target with oxygen ions in an accelerator. The ions are cooled and trapped in a MOT device [52]. Isotopes from ${ }^{208} \mathrm{Fr}$ to ${ }^{221} \mathrm{Fr}$ have been produced and studied. Although all of these isotopes are unstable, some live minutes, for example, $T_{1 / 2}=20 \mathrm{~m}$ for ${ }^{212} \mathrm{Fr}$. The spectrum, including previously unobserved $8 \mathrm{~s}$ and $9 s$ levels, has been established [53, 54]. Precise measurements of lifetimes [55-58] and hyperfine constants $[59,60]$ have been made. Many-body calculations of $E_{\mathrm{PNC}}$ for the $7 s_{1 / 2} \rightarrow 8 s_{1 / 2}$ transition in francium, which have an estimated accuracy of $3 \%$, have also been carried out $[61,62]$.

Recently, a microwave cavity experiment to measure the nuclear spin-dependent PNC amplitude between ground-state hyperfine levels in Fr isotopes has been proposed [63]. A detailed account of the francium PNC research can be found at the website $[64]$.

\subsection{Ytterbium $(Z=70)$}

As pointed out by DeMille [65], the $\left(6 s^{2}\right){ }^{1} S_{0} \rightarrow(6 s 5 d){ }^{3} D_{1}$ PNC transition in atomic $\mathrm{Yb}$ is about 100 times larger than the $6 s \rightarrow 7 s$ transition in cesium. The PNC amplitude for this transition is enhanced by mixing of the final state with the nearby $(6 s 6 p)^{1} P_{1}$ state. Measurements of PNC in ytterbium are particularly interesting since there are seven naturally occurring isotopes ${ }^{168-176} \mathrm{Yb}$ giving the possibility of eliminating uncertainties arising from atomic structure calculations by comparing PNC amplitudes from different isotopes. Two of the isotopes, ${ }^{171} \mathrm{Yb}(I=1 / 2)$ and ${ }^{173} \mathrm{Yb}$ $(I=5 / 2)$, have nonvanishing nuclear spin arising from an unpaired neutron, in contrast with cesium and thallium, where the unpaired nucleon is a proton. Measurements of spin-dependent contributions in ytterbium, therefore, promise new information on the nuclear spin-dependent interaction. It also is interesting to note that the $E 2$ transition ${ }^{1} S_{0} \rightarrow{ }^{3} D_{2}$ has a PNC component that is uniquely sensitive to the spin-dependent part of the weak interaction. The most accurate many-body calculation of the PNC 
amplitude [66] gives

$$
E_{\mathrm{PNC}}=-(1.15 \pm 0.25) \times 10^{-9} \text { i e } a_{0}\left(-Q_{W} / N\right),
$$

accurate to about $20 \%$.

The spectroscopy of the forbidden $\left(6 s^{2}\right){ }^{1} S_{0} \rightarrow(6 s 5 d){ }^{3} D_{1,2}$ transitions, including $E 2$ and $M 1$ transition amplitudes, Stark shifts, isotope shifts and hyperfine structure, has been investigated recently in $[67,68]$. A PNC experiment on ytterbium is underway at Berkeley; details are is available at the website [69].

\subsection{Barium $(Z=56)$ ion}

The possibility of measuring parity nonconservation in a single trapped $\mathrm{Ba}+$ ion was suggested by Fortson [70]. Such a measurement could compete with that for Cs directly; it could also give information on nuclear-spin dependent contributions to $\mathrm{PNC}$ for the odd $\mathrm{Ba}$ isotopes, ${ }^{135} \mathrm{Ba}(6.6 \%$ abundance $)$ and ${ }^{137} \mathrm{Ba}(11.2 \%$ abundance $)$, both of which have nuclear spin $I=3 / 2$. To observe PNC in $\mathrm{Ba}^{+}$, the $E 2$ allowed transition between the $6 s_{1 / 2}$ and $5 d_{3 / 2}$ states is used because of the long lifetime ( $\geq 50$ sec) of the excited $5 d_{3 / 2}$ state. The wavelength of the transition is much larger than the size of the cooled orbit of the trapped ion. Interference between the couplings of the PNC electric dipole matrix element $\left(E_{\mathrm{PNC}} \sim 10^{-11} e a_{0}\right)$ and the $E 2$ matrix element to the electric field of a light wave is the quantity to be measured. Recent progress on the related spectroscopy with a single $\mathrm{Ba}^{+}$ion is reported in [71]. A further discussion of the $\mathrm{Ba}^{+} \mathrm{PNC}$ experiment can be found at the website [22].

\subsection{Dysprosium $(Z=66)$}

Atomic dysprosium has two nearly degenerate levels of opposite parity, $a=\left(4 f^{10} 5 d 6 s\right)[10]$ and $b=\left(4 f^{9} 5 d^{2} 6 s\right)[10]$, at $19797.96 \mathrm{~cm}^{-1}$ above the ground state. Given that dysprosium has two naturally occurring isotopes with nonvanishing nuclear spin ${ }^{161} \mathrm{Dy}$ and ${ }^{163} \mathrm{Dy}$, both with nuclear spin $I=5 / 2$, it is a natural candidate for studying nuclear-spin dependent effects in PNC. A Stark interference experiment to detect the PNC mixing between the states $a$ and $b$ was carried out by Nguyen et al. [72] and gave $\left|H_{W}\right|=|2.3 \pm 2.9 \pm 0.7| \mathrm{Hz}$, where the first error was from systematics and the second was statistical. This result differs substantially from the theoretical result $H_{W}=70(40) \mathrm{Hz}$ obtained in a multi-configuration Dirac-Fock calculation [73].

\subsection{Samarium $(Z=62)$}

The optical rotation parameter $R_{\phi}$ for five $M 1$ transitions between ${ }^{7} F_{J}$ and ${ }^{5} D_{J^{\prime}}$ states in the $\left(4 f 66 s^{2}\right)$ multiplet of samarium was measured by Lucas et al. [74]. The upper state levels are nearly degenerate with levels of opposite parity from the $\left(4 f^{6} 6 s 6 p\right)$ configuration leading to an expected enhancement of the PNC amplitude. The weak interaction matrix elements $\left|H_{W}\right|$ extracted range from 1 to $30 \mathrm{kHz}$. The measured values were found to be one to two orders of magnitude smaller than expected from semi-empirical calculations. 


\section{$5 \quad$ Nuclear Spin Dependent Terms}

For a nucleus with a single unpaired nucleon, the contribution to the PNC interaction Hamiltonian from the axial-vector current of the nuclear quarks is

$$
H^{(2)}=-\frac{G}{\sqrt{2}} c_{2 N} \frac{\kappa-1 / 2}{I(I+1)} \boldsymbol{\alpha} \cdot I \rho_{N}(r)
$$

where, in the extreme shell-model limit, $c_{2 N}$ is the axial-vector coupling constant of the unpaired nucleon. The parameter $\kappa=\mp(I+1 / 2)$ for $I=L \pm 1 / 2$, where $I$ is the nuclear spin and $L$ is the orbital angular momentum of the valence nucleon. $\rho_{N}(r)$ is the valence nucleon density. The standard model gives $c_{2 p}=0.047$ and $c_{2 n}=-0.047$.

A much larger contribution to the spin-dependent PNC interaction arises from the nuclear anapole moment [75]. Weak interactions in the nucleus generate a toroidal current inside the nucleus that couples electromagnetically to the atomic electrons, leading to the interaction

$$
H^{(a)}=\frac{G}{\sqrt{2}} K_{a} \frac{\kappa}{I(I+1)} \boldsymbol{\alpha} \cdot I \rho_{N}(r)
$$

where $K_{a}$ is a coupling constant that depends on the weak processes in the nucleus $[76]$.

A third source of nuclear spin dependent terms arises from interference between the hyperfine nuclear interaction $H_{\mathrm{hf}}$ and $H^{(1)}$. This interference leads to an interaction that can be expressed as

$$
H^{(\mathrm{int})}=\frac{G}{\sqrt{2}} \eta_{\mathrm{int}} \boldsymbol{\alpha} \cdot I \rho_{N}(r) .
$$

The coupling constant $\eta_{\text {int }}$ has been worked out for the cases of cesium and thallium by Bouchiat and Piketty $[77,78]$ to give $\eta_{\text {int }}\left({ }^{133} \mathrm{Cs}\right)=0.0078$ and $\eta_{\text {int }}\left({ }^{205} \mathrm{Tl}\right)=0.044$.

We can combine the three spin-dependent terms as

$$
H^{(\text {s.d. })}=\frac{G}{\sqrt{2}} \eta \boldsymbol{\alpha} \cdot I \rho_{N}(r),
$$

where $\eta=\eta_{2}+\eta_{a}+\eta_{\text {int }}$ can easily be determined in terms of the coupling constants introduced in the above paragraphs. For the cases of cesium and thallium, one finds from the independent-particle model that $\eta_{2}\left({ }^{133} \mathrm{Cs}\right)=0.0151$ and $\eta_{2}\left({ }^{205} \mathrm{Tl}\right)=-0.136$; the corresponding values from a nuclear shell-model calculation [79] are $\eta_{2}\left({ }^{133} \mathrm{Cs}\right)=$ 0.0140 and $\eta_{2}\left({ }^{205} \mathrm{Tl}\right)=-0.127$.

From the Boulder cesium experiment and the Seattle thallium experiment, it is found $[80,81]$ that $\eta_{\exp }\left({ }^{133} \mathrm{Cs}\right)=0.112(16)$ and $\eta_{\exp }\left({ }^{205} \mathrm{Tl}\right)=0.29(40)$. These experimental results lead to the following experimental values for the anapole contributions:

$$
\begin{aligned}
& \eta_{a}\left({ }^{133} \mathrm{Cs}\right)=0.090(16) \\
& \eta_{a}\left({ }^{205} \mathrm{Tl}\right)=0.38(40) .
\end{aligned}
$$

For the case of cesium, weak meson-nucleon coupling constants were inferred from the experimental anapole contributions by Flambaum and Murray [80]. The analysis in Refs. [79, 81], however, indicates that the experimental anapole contributions are not consistent with most general constraints on these coupling constants, which favor a larger value for $\eta_{\exp }$ in cesium and a negative value for thallium. 
The new experiments on cesium, thallium, ytterbium, barium ions and francium will add valuable data needed to resolve the questions concerning the weak nucleonmeson coupling constants.

This work was supported in part by NSF Grant PHY-01-39928.

\section{References}

[1] M. A. Bouchiat and C. Bouchiat, Phys. Lett. 48B, 111 (1974).

[2] O. Laporte, Z. Physik 23, 135 (1924).

[3] M. E. Peshkin and T. Takeuchi, Phys. Rev. D 46, 381 (1992).

[4] M. J. Ramsey-Musolf, Phys. Rev. C 60, 015501 (1999).

[5] C. S. Wood, S. C. Bennett, D. Cho, B. P. Masterson, J. L. Roberts, C. E. Tanner, and C. E. Wieman, Science 275, 1759 (1997).

[6] S. C. Bennett and C. E. Wieman, Phys. Rev. Lett. 82, 2484 (1999).

[7] D. Groom, M. Aguilar-Benitez, C. Amsler, R. Barnett, P. Burchat, C. Carone, C. Caso, G. Conforto, O. Dahl, M. Doser, et al., Euro. Phys. J. C 15, 1+ (2000), URL http://pdg. lbl.gov.

[8] M. J. Ramsey-Musolf, Phys. Rev. D 62, 056009 (2000).

[9] R. Casalbuoni, S. D. Curtis, D. Dominici, and R. Gatto, Phys. Letts. B 460, 135 (1999).

[10] J. L. Rosner, Phys. Rev. D 61, 016006 (2000).

[11] J. Erler and P. Langacker, Phys. Rev. Lett. 84, 212 (2000).

[12] V. Barger and K. Cheung, Phys. Lett. B 480, 149 (2000).

[13] N. H. Edwards, S. J. Phipp, P. E. G. Baird, and S. Nakayama, Phys. Rev. Lett. 74, 2654 (1995).

[14] P. Vetter, D. M. Meekhof, P. K. Majumder, S. K. Lamoreaux, and E. N. Fortson, Phys. Rev. Lett. 74, 2658 (1995).

[15] D. M. Meekhof, P. A. Vetter, P. K. Majumder, S. K. Lamoreaux, and E. N. Fortson, Phys. Rev. A 52, 1895 (1995).

[16] N. H. Edwards, P. E. G. Baird, and S. Nakayama, J. Phys. B 29, 1861 (1996).

[17] M. D. Macpherson, K. P. Zetie, R. B. Warrington, D. N. Stacey, and J. P. Hoare, Phys. Rev. Lett. 67, 2784 (1991).

[18] P. K. Majumder and L. L. Tsai, Phys. Rev. A 60, 267 (1999).

[19] P. G. Kozlov, S. G. Poresv, and W. R. Johnson, Phys. Rev. A 64, 052107 (2001).

[20] A. D. Cronin, R. B. Warrington, S. K. Lamoreaux, and E. N. Fortson, Phys. Rev. Lett. 80, 3719 (1998). 
[21] K.-J. Boller, A. Imamoglu, and S. E. Harris, Phys. Rev. Lett. 66, 2593 (1991).

[22] URL www.phys.washington.edu/ fortson/.

[23] M. A. Bouchiat and C. Bouchiat, J. Phys. (Paris) 35, 899 (1974).

[24] M. A. Bouchiat and C. Bouchiat, J. Phys. (Paris) 36, 493 (1974).

[25] P. S. Drell and E. D. Commins, Phys. Rev. Lett. 53, 968 (1984).

[26] P. S. Drell and E. D. Commins, Phys. Rev. A 32, 2196 (1985).

[27] C. E. Tanner and E. D. Commins, Phys. Rev. Lett. 56, 332 (1986).

[28] M. A. Bouchiat, J. Guena, L. Pottier, and L. Hunter, Phys. Lett. B 134, 463 (1984).

[29] M. L. Wieman and S. L. Gilbert, Phys. Rev. A 34, 792 (1988).

[30] V. A. Dzuba, V. V. Flambaum, and J. S. M. Ginges (2002), arXiv:hep$\mathrm{ph} / 0204134$.

[31] S. A. Blundell, J. Sapirstein, and W. R. Johnson, Phys. Rev. D 45, 1602 (1992).

[32] V. A. Dzuba, V. V. Flambaum, and O. P. Sushkov, Phys. Lett. A 141, 147 (1989).

[33] A. Derevianko, Phys. Rev. Lett. 85, 1618 (2000).

[34] O. P. Sushkov, Phys. Rev. A 63, 042504 (2001).

[35] V. A. Dzuba, C. Harabati, W. R. Johnson, and M. S. Safronova, Phys. Rev. A 63, 044103 (2001).

[36] M. G. Kozlov, S. G. Porsev, and I. I. Tupitsyn, Phys. Rev. Lett. 86, 3260 (2001).

[37] I. Bednyakov, L. Labzowsky, G. Plunien, G. Soff, and V. Karasiev, Phys. Rev. A 61, 012103 (1999).

[38] B. W. Lynn and P. G. H. Sandars, J. Phys. B 27, 1469 (1994).

[39] W. J. Marciano and J. L. Rosner, Phys. Rev. Lett. 65, 2963 (1990).

[40] W. J. Marciano and J. L. Rosner, Phys. Rev. Lett. 68, 898 (1992).

[41] W. J. Marciano and A. Sirlin, Phys. Rev. D 27, 552 (1983).

[42] A. Sirlin, Phys. Rev. D 22, 971 (1980).

[43] W. R. Johnson, I. Bednyakov, and G. Soff, Phys. Rev. Lett. 87, 233001 (2001).

[44] A. I. Milstein and O. P. Sushkov (2001), arXiv:hep-ph/0109257.

[45] M. Y. Kuchiev and V. V. Flambaum (2002), arXiv:hep-ph/0206124.

[46] J. James and P. G. H. Sandars, J. Phys. B 32, 3295 (1999). 
[47] S. J. Pollock and M. C. Welliver, Phys. Lett. B 464, 177 (1999).

[48] A. Derevianko, Phys. Rev. A 65, 052115 (2002).

[49] J. Guéna, D. Chauvat, P. Jacquier, M. Lintz, M. D. Plimmer, and M. A. Bouchiat, J. Opt. Soc. Am. B 14, 271 (1997).

[50] J. Guéna, D. Chauvat, P. Jacquier, M. Lintz, M. D. Plimmer, and M. A. Bouchiat, Quant. Semiclass. Opt. 10, 733 (1998).

[51] E. Jahier, J. Guéna, P. Jacquier, M. Lintz, and M. A. Bouchiat, Eur. Phys. J. D 13, 221 (2001).

[52] J. E. Simsarian, A. Ghosh, G. Gwinner, L. A. Orozco, G. D. Sprouse, and P. A. Voytas, Phys. Rev. Lett. 76, 3522 (1996).

[53] J. E. Simsarian, W. Shi, L. A. Orozco, G. D. Sprouse, and W. Z. Zhao, Opt. Lett. 21, 1939 (1996).

[54] J. E. Simsarian, W. Z. Zhao, L. A. Orozco, and G. D. Sprouse, Phys. Rev. A 59, 195 (1999).

[55] W. Z. Zhao, J. E. Simsarian, L. A. Orozco, W. Shi, and G. D. Sprouse, Phys. Rev. Lett. 78, 4169 (1997).

[56] J. E. Simsarian, L. A. Orozco, G. D. Sprouse, and W. Z. Zhao, Phys. Rev. A 57, 2448 (1998).

[57] J. S. Grossman, L. A. Orozco, M. R. Pearson, and G. D. Sprouse, Physica Scripta T86, 16 (2000).

[58] J. M. Grossman, R. P. Fliller III, L. A. Orozco, M. R. Pearson, and G. D. Sprouse, Phys. Rev. A 62, 062502 (2000).

[59] J. S. Grossman, L. A. Orozco, M. R. Pearson, J. E. Simsarian, G. D. Sprouse, and W. Z. Zhao, Phys. Rev. Lett. 83, 935 (1999).

[60] J. M. Grossman, R. P. Fliller III, T. E. Mehlstaubler, L. A. Orozco, M. R. Pearson, G. D. Sprouse, , and W. Z. Zhao, Phys. Rev. A 62, 052507 (2000).

[61] V. A. Dzuba, V. V. Flambaum, and O. P. Sushkov, Phys. Rev. A 52, 3454 (1995).

[62] M. S. Safronova and W. R. Johnson, Phys. Rev. A 62, 022112 (2000).

[63] S. Aubin, E. Gomez, J. M. Grossman, L. A. Orozco, M. R. Pearson, G. D. Sprouse, and D. P. Demille, in International Conference on Laser Spectroscopy (2001).

[64] URL insti.physics.sunysb.edu/Physics/index.htm.

[65] D. DeMille, Phys. Rev. Lett. 74, 4165 (1995).

[66] S. G. Porsev, Y. G. Rakhlina, and M. G. Kozlov, JETP Lett. 61, 459 (1995). 
[67] C. J. Bowers, D. Budker, S. J. Freedman, G. Gwinner, and J. E. Stalnaker, Phys. Rev. A 59, 3513 (1999).

[68] J. E. Stalnaker, D. Budker, D. P. DeMille, S. J. Freedman, and V. V. Yashchuk (2002), arXiv:physics/0201026.

[69] URL ist-socrates.berkeley.edu/budker/.

[70] N. Fortson, Phys. Rev. Lett. 70, 2383 (1993).

[71] T. W. Koerber, M. H. Schacht, K. R. G. Hendrickson, W. Nagourney, and E. N. Fortson, Phys. Rev. Lett. 88, 143002 (2002).

[72] A.-T. Nguyen, D. Budker, D. DeMille, and M. Zolotorev, Phys. Rev. A 56, 3453 (1997).

[73] V. A. Dzuba, V. V. Flambaum, and M. G. Kozlov, Phys. Rev. A 50, 3812 (1994).

[74] D. M. Lucas, R. B. Warrington, D. N. Stacey, and C. D. Thompson, Phys. Rev. A 58, 3457 (1998).

[75] Y. B. Zeldovich, Sov. Phys. JETP 9, 682 (1959).

[76] V. V. Flambaum, I. B. Khriplovich, and O. P. Sushkov, Phys. Letts. B 146, 367 (1984).

[77] C. Bouchiat and C. A. Piketty, Z. Phys. C 49, 91 (1991).

[78] C. Bouchiat and C. A. Piketty, Phys. Lett. B 269, 195 (1991).

[79] W. C. Haxton, C.-P. Liu, and M. J. Ramsey-Musolf, Phys. Rev. Lett. 86, 5277 (2001).

[80] V. V. Flambaum and D. W. Murray, Phys. Rev. C 56, 1641 (1997).

[81] W. C. Haxton and C. E. Wieman, Ann. Rev. Nucl. Part. Sci. 51, 261 (2001). 